\title{
Using Personality Variables to Predict Cancer and Heart Disease
}

\author{
Manfred Amelang* \\ Department of Psychology, University of Heidelberg, Germany
}

\begin{abstract}
The purpose of this study was to examine the validity of the scales from GrossarthMaticek in the prediction of cancer and coronary heart disease (CHD). Factor analyses, based on responses from a community sample of 5133 subjects between the ages of 40 and 65, showed that the scale measuring Type 1 (disposition for cancer) correlated highly with the scale measuring Type 2 (disposition for CHD) personalities. Both scales had high positive loadings on a factor which had positive markers of neuroticism and depression and negative markers of optimism, sense of coherence, and social support. In two separate case-control studies, the Type 1, 2, and 4 (disposition for health) scales failed to successfully discriminate between groups of healthy individuals and those with a clinical diagnosis of cancer or CHD. Logistic regression analyses using data from the community sample showed that traditional risk factors such as gender, passive smoking, and neuroticism led to significant discrimination between healthy subjects and subjects with cancer or CHD. In contrast the scales from Grossarth-Maticek explained only a small part of the health versus illness variance and have little incremental validity over other traditional health-related personality constructs. (c) 1997 John Wiley \& Sons, Ltd.
\end{abstract}

Eur. J. Pers., 11, 319-342, 1997.

No. of Figures: 2. No. of Tables: 4. No. of References: 65.

\section{INTRODUCTION}

Cancer and heart disease are major health problems throughout the world. In Europe they represent the most frequent causes of death in adult men and women.

\footnotetext{
* Correspondence to: Professor Dr. Manfred Amelang, Psychologisches Institut, Ruprecht-KarlsUniversität Heidelberg, Hauptstrasse 47-51, D-69117 Heidelberg, Germany, Telephone: (+49) 6221 547 328. Fax: (+49) 6221547325.

Contract grant sponsor: Deutsche Forschungsgemeinschaft; Contract grant numbers: Am 37/12-1; Am 37/ $12-2$.
} 
For this reason, intensive research in many disciplines is being directed at identifying potential risk factors for these illnesses. In psychology, a major focus of research has been on the identification of personality factors that may predispose individuals to the development of cancer and coronary heart disease (CHD). This research has repeatedly shown that about 3 per cent of the unique variance in health/illness is explained by personality factors, over and above traditional medical risk factors such as smoking, high blood pressure, high cholesterol levels, and lack of exercise (Contrada, Leventhal, and O'Leary, 1990; Fox, 1988). In sharp contrast, a series of longitudinal, community-based studies by Grossarth-Maticek and Eysenck (see Eysenck, 1991a, for an overview) has identified two personality types that appear to show an extraordinary ability to predict diagnoses of cancer and CHD, respectively. Given the striking discrepancy between the findings of traditional research on personality and health and those of Grossarth-Maticek and Eysenck, their research has been carefully scrutinized and has been the subject of considerable controversy and scepticism (see commentaries in Psychological Inquiry, 1991; Amelang, SchmidtRathjens, and Matthews, 1996).

The purpose of the present article is to re-examine this controversy in light of new data from my ongoing research on personality factors in cancer and heart disease. I begin by presenting a brief selective review of research on personality factors in cancer and CHD to provide a contact for personality researchers who may by unfamiliar with previous work in this area. I then briefly present an overview of the findings of the Grossarth-Maticek studies and the major criticisms of their research. Following this, I present findings from two case-control studies and my initial crosssectional findings from my own large community-based studies using a modified version of Grossarth-Maticek's personality questionnaire. I conclude by attempting to integrate the findings of traditional studies of personality and health, those of Grossarth-Maticek and Eysenck, and the present findings.

\section{RESEARCH ON PERSONALITY AND ILLNESS}

The theory, design, and results of studies of personality and illness have been extensively reviewed by Fox (1988) and Contrada et al. (1990). Here, I present a brief overview of some of the research specifically focused on personality factors in the two diseases studied by Grossarth-Maticek and Eysenck, cancer and CHD.

\section{Cancer}

During the past three decades, many of the investigations into psychosocial risk factors in cancer have focused on two hypotheses: (i) the loss-depression hypothesis and (ii) the 'cancer-prone personality'. According to the first hypothesis, individuals experiencing a higher than expected rate of actual or threatened losses are at higher risk for the development of depression, which, in turn, is expected to be associated with higher rates of cancer. Le Shan (1963), Thomas, Duszynski and Shaffer (1979), and Shekelle, Raynour, Ostfeld, Garron, Bieliauskas, Lui, Maliza and Paul (1981) reported support for this hypothesis, particularly among individuals having personal separations or job instability. On the other hand, the role of depression as a potential risk factor for cancer is less clear. In one prospective longitudinal study by Dattore, 
Shontz, and Coyne (1980) cancer patients showed lower depression scores in comparison with control subjects, whereas in a similar prospective longitudinal study conducted by Shekelle et al. (1981) cancer patients had higher depression scores than control subjects.

As reviewed by Hürny and Adler (1991), research on the hypothesis of the cancer-prone personality has focused on 11 trait clusters: (i) denial and repression, (ii) reduced self-perception, (iii) reduced discharge of emotions, (iv) impaired expression of anger, (v) self-sacrifice and self-accusation, (vi) rigid life-style, conformable to others, (vii) belief in authorities and religiousness, (viii) excessive reality orientation, (ix) flat, vulnerable interpersonal relationships, (x) inhibited sexuality, and (xi) high moral self-concept. Most of the data investigating this hypothesis have been collected in studies of confirmed cancer patients. These investigators have assumed that fundamental structure of personality is formed over a lifetime and is therefore stable and cannot by changed by being diagnosed with cancer. According to this view, the diagnosis of cancer only activates existing traits and coping strategies. The personality trait clusters listed above can generally by considered to represent an increased tendency to repress basic impulses and feelings (Hürny and Adler, 1991).

A prototypical study investigating this hypothesis was conducted by Bahnson and Bahnson (1966) who collected interview and Rorschach test data that provided evidence for massive denial and reaction formation in cancer patients. However, the interviewers were aware of the subject's health status so the possibility that this knowledge influenced the interview process cannot be ruled out. A questionnaire was developed based on these interviews to measure personality factors associated with cancer; however, this questionnaire was only marginally useful in discriminating patients from healthy controls in later studies.

Scherg (1993) reported cross-sectional and longitudinal findings from a sample of 2340 women without evidence of cancer at the onset of the study. Scherg identified several premorbid factors that were associated with breast cancer, among which were a tendency to ignore occurrences related to health and illness and to repress anger. In a 10 year follow-up study of breast cancer patients, Pettingale (1984) found similar results: patients originally categorized as helpless or stoic were more likely to relapse. In contrast, Schwarz (1993), in a prospective study of 230 female patients with suspected mastocarcinoma, found that test and questionnaire results did not predict the malignant versus benign outcome of histological analyses.

As these examples illustrate, the literature provides only weak support for the cancer-prone personality hypothesis. In several of the studies the possibility that the obtained associations may be the consequence of a post hoc analysis of an individual biography (cf. Bilek, Frischenschläger, Reiner and Jakesz, 1988) rather than pre-existing personality factors cannot be ruled out. Several of the studies do not control for confounding risk factors such as smoking and family history of cancer. Few of the longitudinal studies were able to distinguish between different kinds of cancer, implying an equivalent causal pattern of psychological factors underlying, for example, breast and lung cancer. However, given the low base rates of even the most common types of cancer, very large community studies or prospective studies of patient populations who are suspected or known to have cancer at the specific site are necessary to make this distinction. 


\section{Coronary heart disease (CHD)}

Much of the initial interest in the role of personality factors in CHD was stimulated by the work of Friedman and Rosenman $(1959 ; 1974)$ who found that the Type A behaviour pattern (TABP), which they defined as being time-urgent, competitive, and hostile, was associated with a twofold increase in the risk of death from a myocardial infarction. However, subsequent research was far less supportive of this relationship (Booth-Kewley and Friedman, 1987). A part of this discrepancy was attributable to the measurement method: the Structured Interview used by Friedman and Rosenman (1959) showed only modest correlations with the more economical and therefore much more frequently used Jenkins Activity Survey (JAS; Jenkins, 1978). Depending upon the particular subject population and outcome variables that were sampled, the data showed one of two patterns of results: either TABP predicted negative health outcomes only in conjunction with other noxious factors, or TABP predicted negative health outcome independently of the 'classical' risk factors associated with CHD. According to the meta-analysis conducted by Myrtek (1995), the effect size associated with TABP is small, with an estimated population effect size of TABP for CHD of $r=0.009$. Taken together, these findings suggest that the Type A concept has lost much of its validity.

Several authors have sought to isolate the effects of each of the separate components of TABP in relationship to CHD. The constructs of striving (Keltikangas-Järvinen and Räikkönen, 1990), aggression (Wright, 1988), and hostility (Smith, 1992) have each been found to offer significant unique prediction of health outcomes. In their review, Friedman and DiMatteo (1989) reported that the mean correlation between anger/hostility/aggression and health outcomes was $r=0.14$. Based on a meta-analysis of five studies comprising a total of 4867 subjects, Myrtek (1995) estimated a population effect for this relation of 0.02. Although the size of these relationships is again small, it is comparable to the magnitude of effects found in community samples with other medical risk factors. For example, in the well known prospective studies of the Framingham and Western Collaborative Groups addressing heart disease, the correlations between cholesterol and CHD and between smoking and CHD are all under $r=0.15$.

Two observations should be made with respect to the size of these relationships. First, the point-biserial correlation becomes seriously attenuated as the outcome moves away from a 50 per cent event likelihood, which, for negative health events such as myocardial infarction and cancer, are relatively rare occurrences in population studies. Second, in a population of hundreds of millions of people, causal factors of the magnitudes reported refer to thousands of lives yearly. Thus, despite their low magnitude in absolute terms, the risks of heart disease associated with personality factors seem to be of meaningful size and of approximately the same magnitude as those associated with traditional medical risk factors such as cigarette smoking.

\section{The studies of Grossarth-Maticek and Eysenck}

The problems of small effect size, the confounding with traditional medical risk factors, and other methodological problems that have characterized the research on personality and illness are not reported in the prospective studies of GrossarthMaticek and his co-authors. In these studies, the magnitude of the reported effects exceeds those found in any other area of personality research. Both diagnoses of 
cancer and heart disease and deaths from these diseases were predicted with extraordinary accuracy on the basis of a battery of questionnaires. Indeed, the level of predictive success achieved far exceeded that achieved in any previous prospective, aetiological study using psychological or medical risk factors.

To briefly summarize, Grossarth-Maticek in his initial study interviewed a sample of 1353 people living in the small town of Crvenka in the former Yugoslavia. These respondents were assessed using a catalogue of 88 questions which measured, among other things, psychosocial stress and inhibited expression of needs in social settings. Based on a series of analyses, Grossarth-Maticek predicted that 38 persons would fall victim to cancer. The criterion data obtained 10 years later showed that 37 of 38 cases were 'hits'. Grossarth-Maticek (1977) stated that these results indicated a hit rate of 97.3 per cent 'even 100 per cent when the suspected cancer diagnosis is included' (emphasis added). The corresponding hit rate for CHD was 92.1 per cent.

In 1971, Grossarth-Maticek began collecting data in two longitudinal community studies in Heidelberg, Germany. He claims that data have been collected from a total of nearly 30000 subjects, some of whom have been tested repeatedly. The procedures of these studies have not been clearly reported. From the available imprecise descriptions, it appears that subjects completed either (i) a German version of the original questionnaire, (ii) an interview covering the same content, or (iii) both the questionnaire and the interview. The initial results from the Heidelberg studies were nearly as striking as those in the original Crvenka study. Of individuals identified as having a disposition toward cancer, 17.4 per cent of the normal sample and 38.4 per cent of the stressed sample died from cancer, whereas only 1.8 per cent and 7.0 per cent of these two samples, respectively, died from CHD. In contrast, of individuals identified as having a disposition toward CHD, 13.5 per cent of the normal sample and 27.8 per cent of the stressed sample died from CHD, whereas only 5.9 per cent and 2.3 per cent of these sample, respectively, died from cancer (Grossarth-Maticek, Eysenck and Vetter, 1988, pp. 486-487).

Underlying the above studies is a proposed personality classification system of six types. According to Grossarth-Maticek, these six personality types are to varying degrees preprogrammed for health, illness, or social deviance. Type 1 individuals are hypothesized to regard an emotionally highly valued object as the most important condition for their own well being and happiness. Great stress is produced if they fail to achieve nearness to the desired object or person, or fail to be successful in a highly valued occupation. Such failure leads to hopelessness, inactivation, and depression ('understimulation', Grossarth-Maticek et al., 1988). Type 2 individuals are hypothesized to regard an emotionally highly valued person or object as the most important cause for their particular distress and unhappiness. Failing to achieve disengagement from the object causes anger, aggression, and arousal ('overarousal', Grossarth-Maticek et al., 1988). Type 4 individuals show a successful integration of inhibition and excitation processes in the sense of a harmonious balance, a pattern which is expected to be associated with health and autonomy. Grossarth-Maticek also identifies three other types: Type 3 ('Ambivalent-unadjusted ego-centred expression'), Type 5 ('Rational-antiemotional expression'), and Type 6 ('Unadjusted antinormative expression'). Types 3,5 and 6 are hypothesized to be disposed to psychoticism, rationalism, and antisocial tendencies, respectively. Since no research to date has shown an association between Types 3,5 or 6 and any health outcome, they will not be considered in detail below. 
Given the review of personality factors in cancer and heart disease, Types 1 and 2 are of particular interest. Type 1 (hopelessness) is similar to the lossdepression hypothesis in predicting cancer; it is also similar to the (reversed) optimism-health hypothesis (Scheier and Carver, 1985). Type 2 (anger) apparently overlaps with the hostility and aggression components of the TABP. Both types share fixations: Type 1 to reach and Type 2 to avoid specific objects or individuals. Due to the extreme nature of these fixations, both types may be expected to be associated with neuroticism. Most importantly, Type 1 personalities ('Inhibition of self-centred expression') are hypothesized to be more susceptible to cancer, Type 2 personalities ('Barriers in self-centred expression') are hypothesized to be more susceptible to CHD, and Type 4 individuals ('Successful ego-centred expression') are hypothesized to be susceptible to health and autonomy. Grossarth-Maticek et al. (1988) report death rates from cancer and CHD that strongly support these hypotheses. Across three different samples, the unweighted mean death rate from cancer was 34.0 for Type 1 individuals and 4.6 for Type 2 individuals. In contrast, the death rate from CHD was 5.7 for Type 1 and 23.2 for Type 2 individuals.

This research is controversial and readers may reach one of two divergent conclusions. One possibility is that Grossarth-Maticek has made an incredible breakthrough in the measurement of illness-prone personalities and in the prediction of health outcomes. A second possibility is that Grossarth-Maticek's results are dependent on atypical and favourable conditions, methodological errors, and curious data analyses. These criticisms are developed in detail in a special issue of Psychological Inquiry (1991) and by Amelang et al. (1996) and are only briefly outlined here.

\section{Selected points from existing critiques}

(i) The predictions in the Crvenka study were not published until after the criterion data were available. Indeed, Grossarth-Maticek had not yet established his personality types at the time of the original study (Pelosi and Appleby, 1992; 1993).

(ii) The doctor who collected the criterion data (i.e., determining the cause of death from death certificates) in the Crvenka study had full knowledge of the predictions. Grossarth-Maticek and his team collected a large portion of the data in the Heidelberg study.

(iii) In the only paper ${ }^{1}$ providing a full report of the Crvenka study, GrossarthMaticek (1977) estimated the validity of his test to be 'greater than 0.90 to 0.95'. Following the principles of classical test theory (Lord and Novick, 1974), this would mean that the reliability of the predictor variable must be at least 0.95 (index of reliability $r_{\mathrm{tc}}=\sqrt{r}_{\mathrm{tt}}$ ), assuming that no errors occurred in the diagnoses of cancer and heart disease. This assumption of perfect reliability of diagnosis is questionable, particularly when diagnoses are made on the basis of death certificates. Fox (1988) considered results like these to be 'simply unbelievable'.

\footnotetext{
${ }^{1}$ This technical report has not been published, is available only in German, and copies have not been made generally available to researchers.
} 
(iv) There are large inconsistencies in the description of the details of the subject selection process in different publications by Grossarth-Maticek and his colleagues based on the Crvenka study.

(v) The subject loss and refusal rates are exceptionally low in comparison to other longitudinal studies of serious health problems, even those that are well funded and in which substantial monetary resources are devoted to addressing these issues.

(vi) Grossarth-Maticek turned the research data from the Heidelberg studies over to two scientific institutes in December 1982. Van der Ploeg (1991) presents an independent reanalysis comparing the success of Grossarth-Maticek's classifications in predicting deaths from cancer and CHD prior to and following this date. As Van der Ploeg notes 'the psychological and/or somatic data lost most of the predictive power after the moment at which the data were deposited' (italics added).

\section{Some new critical observations}

In addition to these earlier concerns about the work of Grossarth-Maticek and his colleagues, several new issues have more recently arisen.

(vii) Eysenck (1991a) claims to have taken the data in his Tables 1-3 from data presented by Grossarth-Maticek et al. (1988). Yet, there are substantial discrepancies: with the exception of the frequencies in the column marked 'Total', the frequencies do not coincide. Eysenck (1991b) attributes this discrepancy to a different number of unclassifiable cases in the two articles, yet it is the number of subjects allocated to each of the four types which produced the marked discrepancies. Information about the classification of subjects into the Type categories is at the very least inconsistent.

(viii) The procedures and the number of subjects in the Heidelberg studies remain unclear. Grossarth-Maticek constructed several questionnaires containing a total of 450 items. If all subjects answered all questions as claimed by Grossarth-Maticek (1991), then the subjects answered many of the items two or three times because of the overlap among the scales. No comment on these unusual procedures or analyses of the repeated item responses have been reported.

It is also unclear how the items were administered: did subjects complete a paper and pencil questionnaire or were they interviewed? There are several inconsistencies in the procedures that have been reported in various publications as detailed by Amelang and Schmidt-Rathjens (1993; see also Amelang, 1993; Eysenck, 1993, for a response). Grossarth-Maticek, Eysenck and Barrett (1993) more recently reported for the first time that the interviews were conducted in four different ways and that positive results could be obtained for subjects questioned by trained interviewers who were helpful in answering questions and who also conveyed warmth and understanding. Moreover, according to GrossarthMaticek and colleagues, the measure has no predictive validity when completed in any other fashion. Adding post hoc conditions to the description of the basic procedures necessary to collect valid data is certainly an unusual scientific practice, particularly in a programme of research that has now spanned more 
than a quarter of a century. However, it does serve as a kind of final immunization of their work against the criticism expressed in this and other articles.

(ix) Grossarth-Maticek and Eysenck (1995) have introduced a new inventory measuring self-regulation. This construct is similar to the opposite pole of neuroticism, reflecting dimensions such as personal autonomy or independence. The inventory was constructed using questions that were useful in discriminating good health from poor health in past research. In an analysis reporting the results of a 15 year longitudinal study involving over 5000 subjects, the inventory is said to show a very high correlation with mortality rates. Indeed, the predictive validities appear to be even better than those associated with the six Grossarth-Maticek Types. The specific items that comprise the new inventory and the intercorrelations of the new inventory with the six Grossarth-Maticek Types are not reported. A detailed psychometric analysis of the new inventory is promised in a later publication.

\section{Implications}

The above criticisms highlight several of the sources of the uncertainty about the findings of Grossarth-Maticek and his colleagues. The magnitudes of the effect are many times greater than in previous research using similar constructs, the details of the studies are not reported consistently, the details of the data analyses are not reported, and even features of the results of the same study are not reported consistently from one article to the next. Such inconsistency and lack of information, particularly with respect to the procedures and data analyses, makes the exact replication of the Grossarth-Maticek studies impossible. Nonetheless, some kind of replication is necessary to provide additional empirical evidence in the controversy. Within such a study, it is at least possible to investigate those specific features of the studies that have been documented thoroughly and consistently. As an initial step towards this end, I investigated the psychometric properties of the published questionnaires from Grossarth-Maticek and his co-authors. I then looked at the ability of the scales to discriminate healthy people from those diagnosed with cancer or CHD.

\section{PSYCHOMETRIC STUDY}

\section{Method}

\section{Subjects and procedures}

The psychometric study was performed using the baseline data from a large sample of residents of Heidelberg, Germany, who are participating in my ongoing longitudinal study of personality and health. In Germany, all residents are required to register in the city in which they reside. A list of the names and addresses of all residents within the targeted age-range of 40-65 years of age were made available by the city governments of Heidelberg and several neighbouring communities. After eliminating residents with obvious non-German surnames to minimize potential language problems, a random sample of the residents was contacted by telephone and mail and were invited to participate in the study. Subjects were offered compensation of 30 DM for their participation. When consent for participation was provided, the subjects 
were invited to the Psychology Department or a community building where data were collected in small groups. If subjects were unable to come to the University, questionnaires were posted to them. Data were collected from a total of 5133 subjects. There were some statistically significant mean differences on a few of the scales between subjects who completed the scales at the University and those who responded by post. However, in terms of Cohen's (1988) guidelines these statistically significant effects were uniformly very small in magnitude (maximum value of $d$ across all scales 0.13 ) and reflected the large sample size. Of subjects who could be contacted, 56 per cent participated in the study. The response rate may have been decreased by legal requirements for the consent form for the longitudinal study: the consent form mentioned the possibility of later diseases and requested written permission from subjects to collect data on their cause of death.

\section{Measures}

In planning the longitudinal study, I wished to collect data using the GrossarthMaticek questionnaire as well as several other standard personality measures that have been used in the area of personality and health. In prior work (Amelang and Schmidt-Rathjens, 1992), based on a sample of Heidelberg residents, we initially shortened the Grossarth-Maticek scales from 323 to 260 items by eliminating identical items that appeared more than once. We then selected items to represent each of the five Grossarth-Maticek scales based on their item-scale total correlations, resulting in a questionnaire of 140 items. The resulting instrument was named the R(evised)-Scales. Preliminary work based partly on a subsample of early enrollees in the longitudinal study who were given the full Grossarth-Maticek scale (Amelang et al., 1996; Schmidt-Rathjens and Amelang, 1993; SchmidtRathjens, Amelang and Kober, 1994) showed that each of the R-Scales was very highly intercorrelated with the corresponding Grossarth-Maticek scale and was highly stable over a 3 month period $(n=77$; test-retest $r=0.91)$.

In addition to our measures of the five Grossarth-Maticek constructs, we collected data on several published health-related measures that are available in German language versions. These measures included (i) Depression (Depressivitäts-Skala; Von Zerssen, 1976), (ii) LOT Optimism (Life Orientation Test; Scheier and Carver, 1985), (iii) Time Urgency and Perpetual Activation Scale (TUPA; Wright, McCurdy and Rogoll, 1992), (iv) Internal Locus of Control over Diseases (from Fragebogen zur Erfassung gesundheitsbezogener Kontrollüberzeugungen; Ferring and Filipp, 1989), (v) External Locus of Control over Diseases (from Fragebogen zur Erfassung gesundheitsbezogener Kontrollüberzeugungen; Ferring and Filipp, 1989), (vi) Social Support (SOZU K-22; Fydrich, Sommer, Menzel and Höll, 1987), (vii) List of Critical Life Events (13 items from Mummendey, in Filipp, 1981), (viii) Anger In (from StateTrait Anger Expression Inventory-STAXI; Schwenkmezger, Hodapp and Spielberger, 1992), (ix) Anger Control (from STAXI; Schwenkmezger et al., 1992), (x) Anger Out (from STAXI; Schwenkmezger et al., 1992), (xi) Psychoticism (Baumann and Dittrich, 1976), (xii) Neuroticism (from Eysenck Personality Inventory-EPI; Eggert, 1974), (xiii) Extraversion (from EPI; Eggert, 1974), (xiv) Social Desirability (from EPI; Eggert, 1974), (xv) Jealousy (from Buss-Durkee Hostility Inventory; in Kornadt, 1982), (xvi) Irritability (from Buss-Durkee Hostility Inventory; in Kornadt, 1982), (xvii) Aggression (from Saltz-Epstein Inventory; in 
Kornadt, 1982), (xviii) Exaggerated Control (from Way of Life Scale; Wright, Von Bussmann, Freidman, Khoury, Owens and Paris, 1990), (xix) Sense of Coherence I (Antonovsky, 1987), and (xx) Sense of Coherence II (Schmidt-Rathjens, Benz, Van Damme, Feldt and Amelang, 1997). In addition, measures of several other new health related constructs were developed for the longitudinal study. These included stress in the work setting, stress in the non-work setting, active smoking, passive smoking, amount of sleep, amount of relaxation, family history of cancer, and family history of CHD. Finally, the items of the questionnaire developed by Quander-Blaznik (1991) were used.

\section{Results}

I report below for the first time psychometric results on the R-Scales based on the full sample of 5133 subjects. Sample sizes vary slightly from analysis to analysis because of missing data. Alpha coefficients showed that the internal consistency was satisfactory for each of the R-Scale measures of the Grossarth-Maticek types: Type 1 (0.90), Type 2 (0.92), Type 3 (0.83), Type 4 (0.89), Type $5(0.91)$, and Type $6(0.76)$. In contrast to the strikingly different mortality rates of Type 1 and Type 2 individuals reported by Grossarth-Maticek et al. (1988), the correlation between RScales 1 and 2 was $r=0.81$, a value that approaches the reliabilities of the two scales.

A correlation matrix was constructed that included the six R-Scales and the 20 published health-related measures. A principal-components analysis with varimax rotation was conducted; a scree plot of the eigenvalues suggested three factors. The factor loadings are presented in Table 1. R-Scales 1 and 2 both loaded highly on Factor 1. Depression, anger in, and neuroticism also had high positive loadings on this factor, while R-Scale 2, optimism, social support, and sense of coherence had high negative loadings. Additional analyses extracting four, five and six factors showed little change in the composition of Factor 1 which accounted for 53.2 per cent of the variance. Given these results it appears that virtually nothing additional is measured with the Type 1 or Type 2 constructs beyond what is already covered by other well known constructs. In other words, there appears to be little evidence for the claim that the Type 1 and Type 2 scales measure new constructs that could serve as the source of unique predictive validity.

I also examined the three-factor, varimax rotated, solutions when the analyses were conducted separately for (i) males and females, (ii) older and younger subjects divided at the median age of 53, and (iii) subgroups of subjects reporting different health histories. The factor structures for males $(n=2440)$ and females $(n=2623)$ were highly similar. The Spearman rank order correlation between the factor loadings for the two groups were as follows: Factor $1, r_{\mathrm{s}}=0.99$, Factor $2, r_{\mathrm{s}}=0.92$, Factor $3, r_{\mathrm{s}}=0.97$. The factor structures for the younger $(n=2557)$ and older $(n=2461)$ subjects were also highly similar for the first two factors: Factor $1, r_{\mathrm{s}}=0.99$, Factor $2, r_{\mathrm{s}}=0.94$ and Factor $3, r_{\mathrm{s}}=0.75$. Finally, the factor structures were compared for a healthy group of subjects who reported no health complaints $(n=539)$, a group of subjects reporting a current or past history of heart disease (angina, heart attack, heart insufficiency, and combinations thereof) $(n=666)$, and a group reporting a current or past history of cancer $(n=203)$. Once again, the factor loadings were highly similar for Factor 1 (healthy-heart disease, $r_{\mathrm{s}}=0.98$; healthy-cancer, $r_{\mathrm{s}}=0.99$; heart disease-cancer, $r_{\mathrm{s}}=0.98$ ) and somewhat lower, but still similar for Factor 3 (healthy-heart disease $=0.92$, healthy-cancer $=0.88$, and heart disease-cancer, $r_{\mathrm{s}}=0.97$ ). However, 
Table 1. Rotated loadings (varimax) on three factors for the whole sample of participants (due to some missing values, $N=5063$ )

\begin{tabular}{|c|c|c|c|c|}
\hline Scales & Factor 1 & Factor 2 & Factor 3 & $h^{2}$ \\
\hline \multicolumn{5}{|l|}{ R-Scale } \\
\hline 1 Inhibitions & 0.76 & 0.04 & 0.31 & 0.68 \\
\hline 2 Barriers & 0.79 & -0.17 & 0.36 & 0.78 \\
\hline 3 Psychopathology & 0.55 & -0.12 & 0.63 & 0.72 \\
\hline 4 Health/Autonomy & -0.77 & 0.37 & 0.18 & 0.76 \\
\hline 5 Rationalism/Antiemotionality & 0.19 & 0.65 & 0.46 & 0.67 \\
\hline 6 Antisocial Tendencies & 0.41 & -0.21 & 0.59 & 0.56 \\
\hline Depression* & 0.76 & -0.11 & 0.16 & 0.61 \\
\hline LOT $(\text { Optimism })^{\dagger}$ & -0.72 & 0.11 & 0.10 & 0.54 \\
\hline $\begin{array}{l}\text { Time Urgency and Perpetual Activation } \\
\text { (TUPA) }\end{array}$ & 0.24 & -0.04 & 0.68 & 0.52 \\
\hline External Locus of Control over Diseases ${ }^{\S}$ & 0.42 & 0.22 & 0.31 & 0.32 \\
\hline Internal Locus of Control over Diseases & -0.21 & 0.13 & 0.43 & 0.25 \\
\hline Social Support & -0.61 & 0.06 & 0.07 & 0.38 \\
\hline Critical Life Events & 0.03 & -0.26 & 0.01 & 0.07 \\
\hline Anger $\operatorname{In}^{* *}$ & 0.59 & 0.07 & 0.19 & 0.39 \\
\hline Anger Control** & -0.15 & 0.67 & -0.05 & 0.47 \\
\hline Anger Out** & 0.21 & -0.59 & 0.39 & 0.54 \\
\hline Psychoticism ${ }^{\dagger \dagger}$ & 0.28 & 0.08 & 0.07 & 0.09 \\
\hline Neuroticism & 0.67 & -0.34 & 0.24 & 0.62 \\
\hline Extraversion & -0.43 & -0.36 & 0.43 & 0.51 \\
\hline Lie Scale (Social Desirability) & -0.02 & 0.68 & -0.03 & 0.46 \\
\hline Irritability $\$$ & 0.45 & -0.55 & 0.26 & 0.58 \\
\hline Jealousy || $\mid$ & 0.57 & -0.16 & 0.30 & 0.44 \\
\hline Aggression $\uparrow$ & 0.20 & -0.49 & 0.36 & 0.42 \\
\hline Exaggerated Social Control (WOLS) ${ }^{* * *}$ & -0.03 & -0.23 & 0.54 & 0.34 \\
\hline Sense of Coherence I (SOC-I) $)^{\dagger \dagger \dagger}$ & -0.78 & 0.34 & 0.03 & 0.72 \\
\hline Sense of Coherence (SOC-II) & $-\mathbf{0 . 8 3}$ & 0.24 & 0.13 & 0.76 \\
\hline Variance explained & 7.02 & 3.13 & 3.05 & \\
\hline$\%$ variance explained & 53.2 & 23.7 & 23.1 & \\
\hline
\end{tabular}

Note: Numbers in bold indicate loadings $\geqslant 0.50$.

* Depressivatäts-Skala (Depression Scale; Von Zerssen, 1976).

${ }^{\dagger}$ Life Orientation Test (LOT; Scheier and Carver, 1985).

\$ 13 Items from the Time Urgency and Perpetual Activation Scale (TUPA; Wright et al., 1992).

${ }^{\S}$ Fragebogen zur Erfassung gesundheitsbezogener Kontrollüberzeugungen (questionnaire for measuring the locus of control over disease; Ferring and Filipp, 1989).

" Soiale Unterstützung (Social Support, SOZU-K-22; Fydrich et al., 1987).

13 Items from the List of Critical Life Events (Mummendey in Filipp, 1981).

**24 (Trait) Items from the State-Trait anger expression inventory (STAXI; Schwenkmezger et al., 1992).

$\dagger$ Psychoticism Scale (Baumann and Dittrich, 1976).

th Eysenck Personality Inventory (EPI; Eggert, 1974).

$\$$ Irritability: Buss-Durkee Hostility Inventory (in Kornadt, 1982).

|| I Jealousy: Buss-Durkee Hostility Inventory (in Kornadt, 1982).

" Aggression: Saltz-Epstein Inventory (in Kornadt, 1982).

***Way of Life Scale (WOLS; Wright, Von Bussmann et al., 1990)

${ }^{\dagger \dagger}$ Sense of Coherence Scale I (SOC-I; Antonovsky, 1987).

+H. Sense of Coherence Scale II (SOC-II; Schmidt-Rathjens et al., 1997). 
the factor loadings for Factor 2 were far lower: for healthy-heart disease groups, $r_{\mathrm{s}}=0.77$; healthy-cancer, $r_{\mathrm{s}}=0.28$; and for heart disease-cancer, $r_{\mathrm{s}}=0.53$. Although these results do reveal small to substantial differences in the factor loadings for Factors 2 and 3, the loadings for Factor 1 on which the critical R-Scales 1 and 2 load are consistent across gender, age and self-reported health status.

\section{CASE-CONTROL STUDIES: UNIVARIATE COMPARISONS}

I have now performed two case-control studies comparing the responses of patients with cancer or heart disease with healthy controls on R-Scales 1, 2, and 4. Such crosssectional case-control studies cannot replace prospective longitudinal studies, but they do provide preliminary evidence on the association of these R-Scale types and health status. My first case-control studies cannot replace prospective longitudinal studies, but they do provide preliminary evidence on the association of these R-Scale types and health status. My first case-control study (Amelang et al., 1996) used the first cohort of subjects to enrol in the longitudinal study. Groups of subjects were identified who reported actual or previous cancer $(n=58)$, actual or previous CHD $(n=208)$, or no previous history of either of these diseases (control group, $n=1326$ ). The results showed that the cancer group scored higher than the CHD group, who, in turn, scored higher than the control group on R-Scales 1 and 2. The control group tended to score higher than the cancer and CHD groups on R-Scale 2. Recall that Grossarth-Maticek and Eysenck predict that cancer patients will score higher than CHD patients and controls on R-Scale 1, whereas CHD patients will score higher than cancer patients and controls on R-Scale 2. The two new case-control studies below present additional data relevant to this hypothesis.

\section{STUDY 1}

\section{Method}

In Study 1, a total of 292 subjects was recruited. The mean age of the total sample was 57.72 years (range 39-81); males and females did not differ in age. A cancer group was recruited through cancer self-help groups $(n=104)$. The predominant forms of cancer in the males were intestinal and colon cancer and the predominant forms of cancer in females were breast and uterine. A CHD group $(n=53)$ was recruited from training, recovery, and sporting groups. The most common diagnoses were heart attack followed by angina pectoris. In the recruitment of the first two groups a third group $(n=19)$ was identified who had diagnoses of both cancer and CHD. The health status of the three illness groups was confirmed through their medical records. Finally, a fourth group of healthy subjects was identified through snowball sampling in which informants initially referred possible healthy subjects, who, in turn, referred others. Only subjects who reported no current health complaints were included in the study. All subjects in the study completed R-Scales 1, 2, and 4.

\section{Results}

The mean scores of each of the four groups are presented in Figure 1. The data were subjected to a 4 (Illness Group) $\times 2$ (Gender) $\times 2$ (Age) analysis of variance 


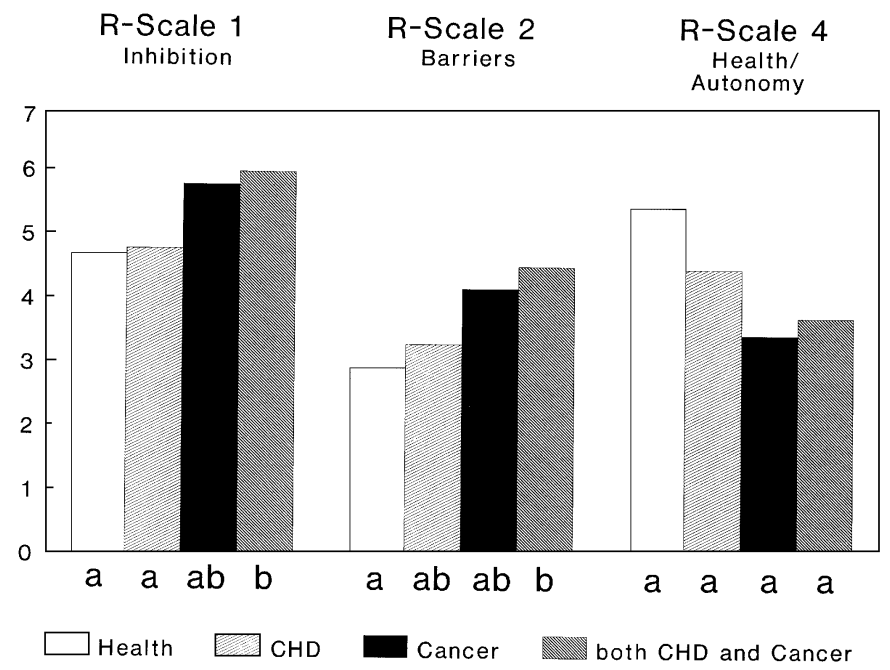

Figure 1. Mean scores of three illness/one health group on R-Scales 1, 2, and 4: CaseControl Study 1. Note: Groups with different subscripts are significantly different by the Newman-Keuls test

separately for each R-Scale. Older subjects were at or above the median age of 57; younger subjects were below 57 . For R-Scale 1 , there were significant effects for both the illness group main effect, $F(3,277)=5.48, p<0.01$, and the illness group $\times$ gender interaction, $F(3,277)=2.66, p<0.05$. A Newman-Keuls test showed that the groups diagnosed with cancer had significantly higher scores than the healthy and the CHD group. The males scored higher than the females within the healthy group, whereas the females scored higher than the males within the two illness groups. For R-Scale 2 , only a main effect for illness group was found, $F(3,277)=4.71, p<0.01$. The Newman-Keuls test showed the group diagnosed with cancer had a significantly higher score than the healthy group. Finally, for R-Scale 2, no effect reached statistical significance. The apparent differences among the illness groups did not attain conventional levels of statistical significance, $F(3,277)=2.03, p=0.11$. Of importance is that no differences between the cancer and the CHD groups were found for any of the R-Scales. Consistent with Grossarth-Maticek's predictions, the cancer group scored higher than the CHD group on R-Scale 1. However, the cancer group also tended to score higher than the CHD group of R-Scale 2, contrary to his prediction. As expected, the healthy group scored lowest on R-Scales 1 and 2, which measure illness-prone personalities, whereas this group showed highest on R-Scale 2, which measures health-prone personality.

\section{STUDY 2}

\section{Method}

Male and female inpatients (total $n=164$ ) were recruited from four hospitals. One group of subjects $(n=50)$ consisted of 50 cancer patients diagnosed with lung, breast, intestinal and colon cancers. A second group $(n=60)$ consisted of CHD patients who 
were diagnosed with myocardial infarction. A third control group $(n=54)$ were patients with orthopaedic complaints. All patients were contacted individually, but filled out the questionnaire privately in the absence of the interviewer. The questionnaire was a 71-item German language questionnaire developed by Grossarth-Maticek (1989) to measure the six Types. With the exception of the addition of one item, it is largely equivalent to the 'Short Interpersonal Reactions Inventory' published by Grossarth-Maticek and Eysenck (1990).

\section{Results}

The mean scores of each of the illness groups on the Type 1, Type 2, and Type 4 Scales are presented in Figure 2 . A series of 3 (Illness Group) $\times 2$ (Gender) analyses of variance were conducted separately for each scale. No significant differences were obtained for the Illness Group or Illness Group $\times$ Gender interaction for any of the scales.

\section{Finer distinctions within disease categories}

Although the above analyses address the overall association of cancer and heart disease with the Grossarth-Maticek Types, distinctions can also be made between types of cancer and types of heart disease. Consequently, in the data base of my longitudinal study, I identified all subjects with a diagnosis of cancer $(n=205)$ and all subjects with a diagnosis of CHD $(n=561)$. For the male cancer patients, the following diagnostic classifications were used: bladder, melanoma, testicular, kidney, combinations, diverse (includes intestinal and colon). For the female cancer patients, the following diagnostic classifications were used: melanoma, breast, uterus, combinations, diverse. For heart disease patients, the diagnostic classifications were angina pectoris, angina plus heart insufficiency, angina plus infarct, angina plus

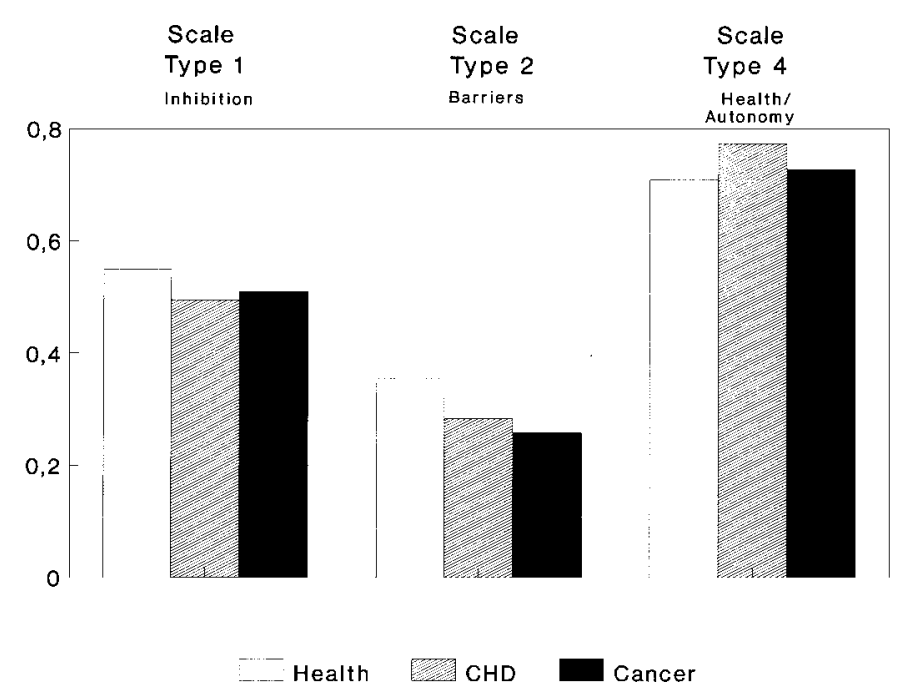

Figure 2. Mean scores of three illness/one health group on R-Scales 1, 2, and 4: CaseControl Study 2 
infarct plus heart insufficiency, heart insufficiency, infarct, and infarct plus heart insufficiency. No cases of infarct or angina plus infarct occurred in females. As in other studies involving community samples, the number of subjects falling in some diagnostic classifications is low.

\section{Results}

I initially performed a series of multivariate analyses of variance (MANOVAs) comparing the diagnostic classifications for cancer and CHD, respectively, on the Grossarth-Maticek and other personality scales described earlier. For the cancer subjects, the MANOVA showed no difference among the six diagnostic classifications for the males, Wilks' lambda $=0.0916, F(130,206.988)=0.99$, ns, and no difference among the five diagnostic classifications for the females, Wilks' lambda $=0.3836$, $F(104,4032.345)=1.06$, ns. For the male CHD subjects, the MANOVA also showed no difference among the seven diagnostic categories, Wilks' lambda $=0.5802, F(156$, $1738.682)=1.08$, ns. However, for the female CHD subjects, a different picture emerged: Wilks' lambda $=0.4763, F(104,7520.117)=1.57, p<0.001$. A series of follow up univariate analyses of variance was performed separately for each of the 26 personality scales. For four of the scales, significant differences were obtained. The means for each of these scales corresponding to the diagnostic categories are presented in Table 2. Newman-Keuls tests showed significant differences between diagnostic categories only in the cases of R-Scale 1 and External Control Over Disease. In both cases, the group with the combination of angina, infarct, and heart sufficiency (i.e., the most severe cases) scored higher than the other groups. Note, however, that the theory of Grossarth-Maticek would expect R-Scale 2 to serve as the relevant personality variable for $\mathrm{CHD}$. Given the absence of a priori predictions about differences between the diagnostic categories and the large number of tests that were performed, these results must be treated cautiously.

\section{CASE-CONTROL STUDIES: MULTIVARIATE COMPARISONS}

One of the central questions for the Grossarth-Maticek measures is whether they have incremental validity: do they offer unique prediction of health outcomes over and above the traditional medical risk factors and personality measures? Given Grossarth-Maticek's theory and previous findings, Type 1 should be an important predictor of the occurrence of CHD. To answer this question, I identified from the data base of my longitudinal community sample described above 533 very healthy individuals who reported no health complaints, 187 subjects who reported cancer, and 654 who reported heart disease. Because of missing data, the number of subjects is reduced slightly in the analyses below.

\section{Results}

In addition to the R-Scale measures of the Grossarth-Maticek types, the other personality variables and medical risk factors described above were included in the data base. Given the large number of predictor variables, I performed two separate stepwise logistic regression analyses. The first analysis contracted the cancer group with the 
Table 2. Frequency of different kinds of CHD and mean scores in selected personality scales for female persons

\begin{tabular}{|c|c|c|c|c|c|c|c|c|}
\hline & $\begin{array}{l}\text { Angina } \\
\text { pectoris }\end{array}$ & $\begin{array}{l}\text { Angina }+ \text { Heart } \\
\text { insufficiency }\end{array}$ & $\begin{array}{l}\text { Angina }+ \\
\text { Infarct }\end{array}$ & $\begin{array}{c}\text { Angina }+ \text { Infarct } \\
+ \text { Heart } \\
\text { insufficiency }\end{array}$ & $\begin{array}{c}\text { Heart } \\
\text { insufficiency }\end{array}$ & Infarct & $\begin{array}{l}\text { Infarct }+ \text { Heart } \\
\text { insufficiency }\end{array}$ & \\
\hline$n$ & 86 & 48 & 0 & 14 & 77 & 0 & 7 & 232 \\
\hline R-Scale $1 M$ & $3.13_{\mathrm{ab}}$ & $3.22_{\mathrm{ab}}$ & & $3.54_{\mathrm{ab}}$ & $3.04_{\mathrm{ab}}$ & & $2.77_{\mathrm{b}}$ & $\begin{array}{c}F(4,224)=2.71 \\
p<0.05\end{array}$ \\
\hline R-Scale $5 M$ & 3.32 & 3.48 & & 3.56 & 2.99 & & 3.29 & $\begin{array}{c}F(4,224)=5.18 \\
p<0.001\end{array}$ \\
\hline Depression $M$ & 0.66 & 0.74 & & 1.05 & 0.67 & & 0.98 & $\begin{array}{c}F(4,224)=2.87 \\
p<0.02\end{array}$ \\
\hline $\begin{array}{l}\text { External } \\
\text { Control over } \\
\text { Diseases } M\end{array}$ & $2.79_{b}$ & $2.69_{b}$ & & $3.31_{\mathrm{a}}$ & $2.55_{\mathrm{b}}$ & & $2.78_{b}$ & $\begin{array}{c}F(4,224)=3.88 \\
p<0.01\end{array}$ \\
\hline
\end{tabular}

Note: Different subscripts indicate significant differences. 
Table 3. Analyses of maximum-likelihood estimates (health $n=519$ versus cancer $n=187$ )

\begin{tabular}{|c|c|c|c|c|c|}
\hline \multirow[b]{2}{*}{ Variable label } & \multirow[b]{2}{*}{ Wald $\chi^{2}$} & \multirow[b]{2}{*}{$\operatorname{Pr}>\chi^{2}$} & \multirow[b]{2}{*}{ Odds ratio } & \multicolumn{2}{|c|}{$\begin{array}{l}\text { Profile likelihood } \\
\text { confidence limits }\end{array}$} \\
\hline & & & & Lower & Upper \\
\hline Intercept & 18.98 & 0.0001 & & & \\
\hline Neuroticism* & 18.68 & 0.0001 & 1.75 & 1.36 & 2.26 \\
\hline Age & 20.55 & 0.0001 & 1.91 & 1.45 & 2.54 \\
\hline Jealousy* & 12.79 & 0.0003 & 2.17 & 1.42 & 3.32 \\
\hline Gender $(0=$ male, $1=$ female $)$ & 3.71 & 0.0540 & 1.51 & 0.99 & 2.30 \\
\hline Life Satisfaction & 5.75 & 0.0165 & 0.77 & 0.62 & 0.95 \\
\hline Repression of Symptoms & 6.68 & 0.0097 & 0.92 & 0.86 & 0.98 \\
\hline Healthy Food ${ }^{\S}$ & 3.99 & 0.0457 & 1.09 & 1.00 & 1.18 \\
\hline Time Ürgency* & 1.19 & 0.2750 & 0.81 & 0.54 & 1.19 \\
\hline Stress after Work ${ }^{\|}$ & 6.19 & 0.0128 & 1.64 & 1.11 & 2.43 \\
\hline Sufficient Sleep & 6.77 & 0.0093 & 2.39 & 1.26 & 4.70 \\
\hline $\begin{array}{l}\text { External Locus of Control } \\
\text { over Diseases* }\end{array}$ & 9.21 & 0.0024 & 1.62 & 1.19 & 2.21 \\
\hline Type 1 (Inhibitions)* & 2.31 & 0.1288 & 0.72 & 0.47 & 1.10 \\
\hline
\end{tabular}

Note: * For explanation see Table 1.

${ }^{\dagger}$ Eight items measuring life satisfaction in different areas.

* One item from Quander-Blaznik (1991).

$\S$ Nine items measuring the degree of healthy food in contrast to unhealthy food.

" Four items measuring stress after work.

๑ One item measuring sufficient versus insufficient duration of sleep.

Association of predicted probabilities and observed responses: Concordant $=78.3 \%$; Sommer's $D=0.569$; $\gamma=0.570 ; \tau-\mathrm{a}=0.222 ; c=0.784$; goodness-of-fit statistic (Hosmer and Lemeshow) $=12979$ with $8 \mathrm{DF}$ $(p=0.1126)$.

healthy group. I initially chose R-Scale 1 and the other personality variables and medical risk factors as candidate predictors. The final model, which is shown in Table 3, showed that the best predictors of cancer versus healthy status were neuroticism, age, jealousy, and sufficient sleep. Note that R-Scale 1, the theoretically relevant predictor, was not retained and did not contribute significantly to the model. When R-Scale 2 is substituted for R-Scale 1, it also does not contribute significantly to the model.

The second analysis contrasted the CHD group with the healthy group. Here, RScale 2 and the other personality variables and medical risk factors were used as candidate predictors. As shown in Table 4, age, neuroticism, and jealousy are strong predictors; however, stress induced through work overload was also a very strong contributor. Type 2 does make a significant contribution, as predicted, but the amount of unique contribution is small in terms of the goodness-of-fit statistic. Consistent with the high correlation between the two scales, if R-Scale 2 is replaced by R-Scale 1, there is little difference in the final equation.

\section{DISCUSSION}

The present results provide little support for the claim that the Grossarth-Maticek personality scales strongly discriminate between individuals with cancer, CHD, and healthy individuals. Amelang et al. (1996) reached the same conclusion based on the 
Table 4. Analyses of maximum-likelihood estimates (health $n=533$ versus CHD $n=654$ )

\begin{tabular}{|c|c|c|c|c|c|}
\hline \multirow[b]{2}{*}{ Variable label } & \multirow[b]{2}{*}{ Wald $\chi^{2}$} & \multirow[b]{2}{*}{$\operatorname{Pr}>\chi^{2}$} & \multirow[b]{2}{*}{ Odds ratio } & \multicolumn{2}{|c|}{$\begin{array}{l}\text { Profile likelihood } \\
\text { confidence limits }\end{array}$} \\
\hline & & & & Lower & Upper \\
\hline Intercept & 170.16 & 0.0001 & & & \\
\hline Age & 142.04 & 0.0001 & 4.14 & 3.29 & 5.26 \\
\hline Neuroticism* & 36.72 & 0.0001 & 2.06 & 1.64 & 2.61 \\
\hline Stress by Workload ${ }^{\dagger}$ & 27.60 & 0.0001 & 63.30 & 13.84 & 306.79 \\
\hline Jealousy I (low vs all other)* & 3.10 & 0.0784 & 0.74 & 0.53 & 1.04 \\
\hline Jealousy II (high vs all other)*§ & 5.17 & 0.0229 & 2.96 & 1.22 & 8.05 \\
\hline $\begin{array}{l}\text { Aggression I (low vs all } \\
\text { other) }^{* t}\end{array}$ & 4.85 & 0.0277 & 1.44 & 1.04 & 1.98 \\
\hline $\begin{array}{l}\text { Aggression II (high vs all } \\
\text { other)*§ }\end{array}$ & 3.09 & 0.0790 & 0.57 & 0.30 & 1.07 \\
\hline Critical Life Events* & 5.41 & 0.0200 & 1.51 & 1.07 & 2.14 \\
\hline Type 5 (Rationalism)* & 6.21 & 0.0127 & 1.36 & 1.07 & 1.73 \\
\hline CHD in the Family & 5.24 & 0.0221 & 1.42 & 1.05 & 1.92 \\
\hline Repression of Symptoms & 3.48 & 0.0623 & 0.95 & 0.90 & 1.00 \\
\hline Passive Smoking** & 9.45 & 0.0021 & 1.42 & 1.05 & 1.92 \\
\hline Gender $(0=$ male, $1=$ female $)$ & 6.42 & 0.0113 & 0.66 & 0.48 & 0.91 \\
\hline Type 2 (Barriers)* & 9.92 & 0.0016 & 1.59 & 1.19 & 2.13 \\
\hline
\end{tabular}

Note: * For explanation see Table 1.

$\dagger 22$ items measuring stress from the job.

\$ For reasons of computation Jealously and Aggression were coded as dummy variables.

$\$$ A grouping of low versus all other scores.

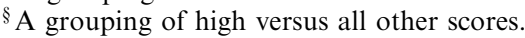

Occurrence of CHD in the family no/yes.

'One item from Quander-Blaznik (1991).

** One item coded no/yes.

For 'low' and 'high', respectively, a 30 per cent criterion was applied.

Association of predicted probabilities and observed responses: Concordant $=85.6 \%$; Sommer's $D=0.713$; $\gamma=0.714 ; \tau-\mathrm{a}=0.353 ; c=0.857$; goodness-of-fit statistic (Hosmer and Lemeshow) $=14337$ with $8 \mathrm{DF}$ $(p=0.9938)$.

far smaller sample of initial enrollees into the community sample. Now, based on the data from the full community sample of over 5000 persons, the results show that the magnitude of the correlation between R-Scales 1 and 2 is so high that they can not be differentiated following correction for attenuation due to measurement error, and that both constructs are highly correlated with neuroticism and depression. Based on such evidence, it is difficult to understand how the strikingly different mortality rates of Type 1 and Type 2 individuals could have been obtained.

The second source of new evidence comes from the results of the two case-control studies conducted using clinically diagnosed cancer and CHD patients. In Study 1, the patients were tested with the R-Scales, which are more reliable, but highly correlated with the original Grossarth-Maticek (1989) scales; in study 2, the patients were tested with these original Grossarth-Maticek scales. The test administration procedures also differed in the two studies (group testing in Study 1; individual testing in Study 2). Nonetheless, little support for the set of Grossarth-Maticek hypotheses was found in either study. In Study 1, significant mean differences were found on the Grossarth-Maticek scales between the cancer, CHD, and healthy 
groups. Consistent with the Grossarth-Maticek predictions, the cancer group had significantly higher scores than the healthy and CHD groups on R-Scale 1 (disposition to cancer). However, contrary to the predictions, the cancer group also had significantly higher scores than the healthy group on R-Scale 2 (disposition to CHD). In Study 2, no significant differences were found among the groups on the Grossarth-Maticek scales. Taken together, the results of the two case-control studies provide little support for the Grossarth-Maticek hypotheses.

The third source of new empirical evidence comes from the logistic regression analyses of the community sample. No significant contribution of the two R-Scales to the discrimination between the cancer and healthy groups was found. Consistent with the Grossarth-Maticek hypotheses, there was a significant contribution of RScale 2 to the discrimination of the CHD and the healthy groups. However, contrary to the hypotheses, the same amount of variance in the discrimination of the CHD and the healthy groups was found when R-Scale 1 was substituted for R-Scale 2. Even more importantly, the neuroticism scale and several other well standardized personality variables explained a considerably larger proportion of the health-illness variance in the logistic regression analysis than did the R-Scales.

There is a small discrepancy in the results of the case-control studies in which the physician diagnosis was used as the criterion for the cancer and CHD groups and the community sample in which diagnoses were self-reported. Several of the effects were statistically significant in the community sample, but not in the case-control studies. These small discrepancies may result from the larger sample size and greater statistical power in the community sample, or they may be the result of the 'softer' quality of the self-report as compared to the clinical diagnosis of illness. Other research suggests that neuroticism may play a role in self-reports of illness (Costa and McCrae, 1987), although this influence is probably lessened in reports of more serious illness such as cancer and CHD.

Taken together, the present studies based on the R-Scale and the original scale measurements of the Grossarth-Maticek types provide little support for GrossarthMaticek's theory. However, as in any study in which non-significant results are obtained, attention must be given to several issues of measurement and methodology. One alternative interpretation is that the R-Scales did not measure the same constructs as the original scales of Grossarth-Maticek. Elsewhere, Schmidt-Rathjens et al. (1994) present data showing that the correlations between each of the R-Scales and the corresponding original Grossarth-Maticek scale are all greater than 0.90 . The present psychometric study also showed that the internal consistencies of R-Scales 1 and 2, the focal scales of interest, were also at least 0.90 . However, in previous work (Amelang and Schmidt-Rathjens, 1993), we found that the correlations between the original Grossarth-Maticek scales measuring Types 1 and 2 ranged between 0.53 and 0.79 , whereas this correlation was 0.81 in the full community sample. This increase in the correlation between Types 1 and 2 measured with the R-Scales may be a result of their increased reliability. Alternatively, the item selection process (i.e., deleting redundant items and reducing the number of total items on the basis of item-total correlations) may have artifactually increased this correlation. No data have been presented by Grossarth-Maticek on the magnitude of this correlation in any published report of the longitudinal study in Crvenka in the former Yugoslavia. Nor have the reports of the more recently developed measures of these Types included these correlations (Grossarth-Maticek, 1989; Grossarth-Maticek and Eysenck, 
1995). One item of data is available in the article by Grossarth-Maticek and Eysenck (1990): a factor analysis indicates that the Type 1 and Type 2 scales both loaded very highly together on one factor, as they did in the present psychometric study, suggesting that Types 1 and 2 are also very highly correlated in the study of Grossarth-Maticek and Eysenck. In any event, it seems improbable that this higher correlation between R-Scales 1 and 2 had a strong influence on the validity of these scales in predicting the external criteria of cancer and CHD. Recall also that in CaseControl Study 2 where the original Grossarth-Maticek scales were used, no mean differences were found among the three health/illness groups. Relatedly, it is possible that the factor structure of the scale changed when the scales were translated from Serbo-Croatian into German (and English). This issue cannot be addressed directly because Grossarth-Maticek has not published factor analyses of either the SerboCroatian or the German language versions of his scale. This argument is weakened, however, because Grossarth-Maticek (1989) originally reported strong prospective prediction of cancer and heart disease in his German study, although this relationship vanished after the data were deposited with two scientific institutes (Van der Ploeg, 1991).

Another consideration in the interpretation of negative results is the conditions of data collection. Contrary to the recent new pronouncement of Grossarth-Maticek et al. (1993), I did not have interviewers who established 'warmth and empathy' before beginning the interview. On the other hand, the questionnaires were administered individually and in groups in different studies, with little resulting difference in terms of either the mean scores or the covariance matrices. In addition, comparisons of different subgroups - male versus female, younger versus older, and even cancer versus CHD versus healthy groups - showed little difference in either the means of the nature of Factor 1 on which measures of both Types 1 and 2 loaded highly. Such findings attest to the stability of the results. In contrast, I know of no other area of research in which the change from an interview to a carefully constructed questionnaire measuring the same construct leads to a change from near-perfect prediction to near-zero prediction.

The pattern of relationships among the variables included in the community sample conformed to general expectations. The principal-component analyses showed meaningful factors in which, for example, neuroticism and depression showed high positive loadings, whereas optimism and sense of coherence showed high negative loadings. Extraversion, one of the markers of the Big Five, loaded on another factor together with components related to the Type A Behaviour Pattern. Many aspects of the results of the factor analysis are consistent with those of Marshall, Wortman, Vickers, Kusulas and Hervig (1994) who concluded that 'most health-relevant dimensions and scales appear to be mixtures of broad personality domains' (p.278). At the same time, my principal-components analysis of a representative community sample of middle-aged German males and females found that measures of optimism versus neuroticism and inhibition tended to load on one factor, whereas their principal-axis factor analysis of two samples of young and healthy male military recruits showed greater differentiation between optimism and inhibition. Both analyses reported a second factor related to anger expression. In addition to the obvious differences in nature of the sample, the variables measured in the two investigations differed substantially so that exact correspondence of the two factor analyses should not be expected (Gorsuch, 1983). 
The logistic regression analyses showed that several of the expected personality and medical risk variables successfully discriminated healthy individuals from those with cancer and healthy individuals from those with CHD. The finding that personality variables provided better discrimination between healthy individuals and those with CHD than between healthy individuals and those with cancer is also consistent with previous research. Note, however, that the larger number of individuals who reported CHD relative to those who reported cancer in the community study may have also contributed to this finding.

Finally, it is important to note that all of the relationships I reported between the personality scales and the measures of health/illness are based on cross-sectional data. It is conceivable that the 'real validity' of the Grossarth-Maticek scales will only appear in longitudinal prospective designs similar to those upon which Grossarth-Maticek's astonishing results are based. Therefore, one more critical test is yet to come concerning personality and illness. The data from the first, 10 year follow up measurement wave of my community sample will soon be available.

\section{ACKNOWLEDGEMENTS}

I am deeply indebted to Stephen G. West whose constructive comments, insight into methodological considerations, and help in organization and editing contributed greatly to the final form of this manuscript. I would like to thank Kimberly Feldt for her assistance in writing the first English language draft of this paper. I would also like to express my gratitude to the two anonymous reviewers and guest editor Sarah Hampson. I thank Annette Scheurer, Alexandra Dorwarth and Sabine Rubi for collecting the data and computing the statistical analyses for the case-control studies. Finally, I thank Safir Yousfi for completing the statistical analyses of the data from the community sample. The research reported in this article was supported by grants Am 37/12-1 and -2 from the Deutsche Forschungsgemeinschaft.

\section{REFERENCES}

Amelang, M. (1993). 'What really happened in Crvenka and Heidelberg?', Zeitschrift für Gesundheitpsychologie, 1: 191-196.

Amelang, M. and Schmidt-Rathjens, C. (1992). 'Psychometric properties of modified Grossarth-Maticek and Eysenck inventories', Psychological Reports, 71: 1251-1263.

Amelang, M. and Schmidt-Rathjens, C. (1993). 'Persönlichkeit, Streß und Krankheit: Untersuchungen zu den psychometrischen Gütekriterien der Krankheitsprädiktoren von Grossarth-Maticek und Eysenck' ['Personality, stress and illness: examining the psychometric properties of the illness predictors from Grossarth-Maticek and Eysenck'], Zeitschrift für Gesundheitspsychologie, 1: 160-182.

Amelang, M., Schmidt-Rathjens, C. and Matthews, G. (1996). 'Personality, cancer and coronary heart disease: further evidence on a controversial issue', British Journal of Health Psychology, 1: 191-205.

Antonovsky, A. (1987). Unraveling the Mystery of Health, Jossey Bass, San Francisco, CA.

Bahnson, C. B. and Bahnson, M. B. (1966). 'The role of the ego defenses: denial and repression in the etiology of malignant neoplasms', Annals of the New York Academy of Sciences, 125: 827-845.

Baumann, U. and Dittrich, A. (1976). 'Überprüfung der Fragebogendimension P (Psychotizismus) im Vergleich zu Extraversion und Neurotizismus' ['Testing the 
questionnaire dimension $\mathrm{P}$ (psychoticism) in comparison to extraversion and neuroticism'], Zeitschrift für Klinische Psychologie, 5: 1-22.

Bilek, P., Frischenschläger, O., Reiner, G. and Jakesz, R. (1988). 'Einschätzungen der Malignität/Benignität einer neoplastischen Veränderung der Brust mit Hilfe von psychosozialen Parametern bei Patientinnen einer Brustambulanz' ['Estimations of malignancy/benignity of neoplastic breast surgery for outpatients with repect to psychosocial parameters'], Psychotherapie-Psychosomatik-Medizinische Psychologie, 38: 420-424.

Booth-Kewley, S. and Friedman, H. S. (1987). 'Psychosocial predictors of heart disease: a quantitative review', Psychological Bulletin, 101: 343-362.

Cohen, J. (1988). Statistical Power Analysis for the Behavioral Sciences, 2nd edn, Erlbaum, Hillsdale, NJ.

Contrada, R. J., Leventhal, H. and O'Leary, A. (1990). 'Personality and health'. In: Pervin, L. A. (ed), Handbook of Personality: Theory and Research, pp.638-669, Guilford, New York.

Costa, P. T., Jr. and McCrae, R. R. (1987). 'Neuroticism, somatic complaints, and disease: is the bark worse than the bite?', Journal of Personality, 55: 299-316.

Dattore, P. J., Shontz, F. C. and Coyne, L. (1980). 'Premorbid personality differentation of cancer and noncancer groups: a test of the hypothesis of cancer proneness', Journal of Consulting and Clinical Psychology, 48: 388-394.

Eggert, D. (1974). Eysenck-Persönlichkeits-Inventar (EPI) [Eysenck's Personality Inventory], Hogrefe, Göttingen.

Eysenck, H. J. (1991a). 'Personality, stress, and disease: an interactionist perpective', Psychological Inquiry, 2: 221-232.

Eysenck, H. J. (1991b). 'Reply to criticisms of the Grossarth-Maticek studies', Psychological Inquiry, 2: 297-323.

Eysenck, H. J. (1992). 'Psychosocial factors, cancer and ischaemic heart disease', British Medical Journal, 305: 457-459.

Eysenck, H. J. (1993). 'Personality, stress and disease: the Grossarth-Maticek contribution', Zeitschrift für Gesundheitspsychologie, 1: 183-190.

Ferring, D. and Filipp, S. H. (1989). 'Der Fragebogen zur Erfassung gesundheitsbezogener Kontrollüberzeugungen (FEGK)' ['Questionnnaire for measuring the locus of control over diseases'], Zeitschrift für Klinische Pscyhologie, 28: 285-289.

Filipp, S. H. (ed) (1981). Kritische Lebensereignisse [Critical Life Events], Urban and Schwarzenberg, Munich.

Fox, B. H. (1988). 'Psychogenic factors in cancer, especially its incidence'. In: Maes, S., Spielberger, C. D., Defares, T. B. and Sarason, I. G. (eds), Topics in Health Psychology, pp. 37-55, Wiley, London.

Friedman, H. S. and Di Matteo, M. R. (1989). Health Psychology, Prentice-Hall, New York.

Friedman, M and Rosenman, R. H. (1959). 'Association of specific overt behavior pattern with blood and cardiovascular findings: blood cholesterol level, blood clotting time, incidence of arcus senilis, and clinical coronary artery disease', Journal of the American Medical Association, 169: 1286-1296.

Friedman, M. and Rosenman, R. H. (1974). Type A Behavior and your Heart, Knopf, New York.

Fydrich, T., Sommer, G., Menzel, G. and Höll, B. (1987). 'Fragebogen zur sozialen Unterstützung (Kurzform; SOZU-K-22)' ['Questionnaire to measure social support (short version)'], Zeitschrift für Klinische Psychologie, 16: 434-436.

Gorsuch, R. L. (1983). Factor Analysis, 2nd edn, Erlbaum, Hillsdale, NJ.

Grossarth-Maticek, R. (1977). Sozialwissenschaftliche Aspekte in der Ätiologie organischer Erkrankungen-Konzeption, Methode und Ergebnisse einer prospektiven Studie [Social Scientific Aspects in the Etiology of Organic Diseases - Conception, Method and Results of a Prospective Study], Veröffentilichung im Rahmen des interdisziplinären Forschungsprojecktes sozialwissenschaftlicher Onkologie, Heidelberg.

Grossarth-Maticek, R. (1989). 'Disposition, Exposition, Verhaltensmuster, Organvorschädigung und Stimulierung des zentralen Nervensystems in der Ätiologie des Bronchial-, Magen-, und Leberkarzinoms' ['Disposition, exposition, behaviour, predamage 
of organs and stimulation of the central nervous system in the etiology of lung, stomach and liver cancer'], Deutsche Zeitschrift für Onkologie, 21: 62-77.

Grossarth-Maticek, R. (1991). Cited in: Eysenck, H. J. (1991b). 'Reply to criticism of the Grossarth-Maticek studies', Psychological Inquiry, 2: 297-323.

Grossarth-Maticek, R. and Eysenck, H. J. (1990). 'Personality, stress and disease: Description and validation of a new inventory', Psychological Reports, 66: 355-373.

Grossarth-Maticek, R. and Eysenck, H. J. (1995). 'Self-regulations and mortality from cancer, coronary heart disease and other causes: a prospective study', Personality and Individual Differences, 19: 781-795.

Grossarth-Maticek, R., Eysenck, H. J. and Barrett, P. (1993). 'Prediction of cancer and coronary heart disease as a function of method of questionnaire administration', Psychological Reports, 73: 943-959.

Grossarth-Maticek, R., Eysenck, H. J. and Vetter, H. (1988). 'Personality type, smoking habit and their interaction on predictors of cancer and coronary heart disease', Personality and Individual Differences, 9: 479-495.

Hürny, C. and Adler, R. (1991). 'Psycho-onkologische Forschung' ['Psycho-oncological Research']. In: Meerwein, F. (ed), Einführung in die Psycho-Onkologie [Introduction to Psycho-Oncology], Huber, Bern.

Jenkins, C. D. (1978). 'A comparative review of the interview and questionnaire methods in the assessment of the coronary-prone behavior pattern'. In: Dembroski, T. M., Weiss, S. M., Shields, J. L., Haynes, S. and Feinleib, M. (eds), Coronary Prone Behaviors, pp. 71-88, Springer, New York.

Keltikangas-Järvinen, L. and Räikkönen, K. (1990). 'Type A factors as predictors of somatic risk factors of coronary heart disease in young Finns - a six-year follow-up study', Journal of Psychosomatic Research, 34: 89-97.

Kornadt, H. J. (1982). Aggressionsmotiv und Aggressionshemmung [Motivation and Inhibition of Aggression], Vol. 2, Huber, Bern.

Le Shan, L. (1963). 'Untersuchungen zur Persönlichkeit der Krebskranken' ['Experiments investigating the personality of cancer patients'], Zeitschrift für Psychosomatische Medizin und Psychoanalyse, 9: 246-253.

Lord, F. M. and Novick, M. R. (1974). Statistical Theories of Mental Test Scores, 2nd edn, Addison-Wesley, Reading, MA.

Marshall, G. N., Wortman, C. B., Vickers, R. R., Jr., Kusulas, J. W. and Hervig, L. K. (1994). 'The five-factor model of personality as a framework for personality-health research', Journal of Personality and Social Psychology, 67: 278-286.

Myrtek, M. (1995). 'Type A behavior pattern, personality factors, disease, and psychological reactivity: a meta-analytic update', Personality and Individual Differences, 18: 491-502.

Pelosi, A. J. and Appleby, L. (1992). 'Psychological influences on cancer and ischaemic heart disease', British Medical Journal, 303: 1295-1298.

Pelosi, A. J. and Appleby, L. (1993). 'Personality and fatal diseases', British Medical Journal, 306: $1666-1667$.

Pettingale, K. W. (1984). 'Coping and cancer prognosis', Journal of Psychosomatic Research, 28: 363-364.

Quander-Blaznik, J. (1991). 'Personality as a predictor of lung cancer: a replication', Personality and Individual Differences, 12: 125-130.

Scheier, M. F. and Carver, C. S. (1985). 'Optimism, coping and health: assessment and implications of generalized outcome expectancies', Health Psychology, 4: 219-247.

Scherg, H. (1993). 'Psychosoziale Faktoren des Mammakarzinoms und anderer Karzinome bei retrospektiver und prospektiver Betrachtungswise' ['Psychosocial factors in mastocarcinoma and other cancers in retrospective and prospective context']. In: Muthny, F. A. and Haag, G. (eds), Onkologie im psychosozialen Kontext [Oncology in the Psychosocial Context], pp. 27-37, Asanger, Heidelberg.

Schmidt-Rathjens, C. and Amelang, M. (1993). 'Psychometrische Gütekriterien und Persönlichkeits-Korrelate der Krankheitsprädiktoren von Grossarth-Maticek und Eysenck' ['Psychometric properties and personality correlates of illness predictors from Grossarth-Maticek and Eysenck'], Diagnostica, 39: 281-298. 
Schmidt-Rathjens, C., Amelang, M. and Kober, G. (1994). 'Disposition und Verhaltensmuster als potentielle Faktoren bei der Krebs- und Infarktgenese: Untersuchungen zu den psychometrischen Gütekriterien eines Fragebogens von Grossarth-Maticek' ['Disposition and behaviour patterns as potential factors for the onset of cancer and infarct: studies examining the psychometric properties of the questionnaire from Grossarth-Maticek'], Deutsche Zeitschrift für Onkologie, 26: 59-64.

Schmidt-Rathjens, C., Benz, D., Van Damme, D., Feldt, K. and Amelang, M. (1997). 'Über zwiespältige Erfahrungen mit Fragebögen zum Kohärenzsinn' ['Discordant experiences with questionnaires measuring Sense of Coherence'], Diagnostica, in press.

Schwarz, R. (1993). “"Krebspersönlichkeit”-Ursache oder Folge der Krebserkrankung?' [" "Cancer personality"-Cause or consequence of cancer?']. In: Muthny, F. A. and Haag, G. (Eds), Onkologie im psychosozialen Kontext [Oncology in the Psychosocial Context], pp. 1-26, Asanger, Heidelberg.

Schwenkmezger, P., Hodapp, V. and Spielberger, C. D. (1992). Das State-TraitÄrgerausdrucks-Inventar (STAXI) [The State-Trait-Anger Expression Inventory], Huber, Bern.

Shekelle, R. B., Raynour, W. J., Jr., Ostfeld, A. M., Garron, D. C., Bieliauskas, L. A., Liu, S. C., Maliza, C. and Paul, O. (1981). 'Psychological depression and 17-year risk of death from cancer', Psychosomatic Medicine, 43: 117-125.

Smith, T. W. (1992). 'Hostility and health', Health Psychology, 11: 139-150.

Thomas, C. B., Duszynski, K. R. and Shaffer, J. W. (1979). 'Family attitudes reported in youth as potential predictors of cancer', Psychosomatic Medicine, 41: 287-302.

Van der Ploeg, H. M. (1991). 'What a wonderful world it would be: a reanalysis of some of the work of Grossarth-Maticek', Psychological Inquiry, 2: 280-285.

Von Zerssen, D. (1976). Die Paranoid-Depressivitäts-Skala [The Paranoid-Depressive Scale], Beltz-Test, Weinheim.

Wright, L. (1988). 'The Type A behavior pattern and coronary heart disease', American Psychologist, 43: 2-14.

Wright, L., McCurdy, S. and Rogoll, G. (1992) 'The TUPA scale: a self-report measure for the Type A subcomponent of time urgency and perpetual activation', unpublished manuscript, Univerisity of Oklahoma.

Wright, L., Von Bussmann, K., Freidman, A. Khoury, M., Owens, F. and Paris, W. (1990). 'Exaggerated social control and its relationship to the Type A behavior pattern', Journal of Research in Personality, 24: 258-269. 
Copyright of European Journal of Personality is the property of John Wiley \& Sons Inc. and its content may not be copied or emailed to multiple sites or posted to a listserv without the copyright holder's express written permission. However, users may print, download, or email articles for individual use. 